\title{
Femtosecond spectroscopy of acoustic frequency combs in the 100-GHz frequency range in $\mathrm{Al} / \mathrm{Si}$ membranes
}

\author{
Martin Grossmann, ${ }^{1, *}$ Matthias Klingele,${ }^{1}$ Patricia Scheel,${ }^{1}$ Oliver Ristow, ${ }^{1}$ Mike Hettich, ${ }^{1}$ Chuan He, ${ }^{1}$ Reimar Waitz, \\ Martin Schubert, ${ }^{1}$ Axel Bruchhausen, ${ }^{2}$ Vitalyi Gusev, ${ }^{3}$ Elke Scheer, ${ }^{1}$ and Thomas Dekorsy ${ }^{1}$ \\ ${ }^{1}$ Department of Physics, University of Konstanz, Germany \\ ${ }^{2}$ Instituto Balseiro \& Centro Atómico Bariloche (CNEA) and CONICET, Argentina \\ ${ }^{3}$ IMMM, UMR-CNRS 6283, Université du Maine, Av. O. Messiaen, 72085 Le Mans, France
}

(Received 28 January 2013; revised manuscript received 26 July 2013; published 8 November 2013)

\begin{abstract}
Acoustic frequency combs are optically excited and detected in silicon membranes covered with thin aluminum layers by femtosecond pump-probe spectroscopy. The various frequency combs consist of 11 up to 45 modes ranging in frequency from 10 up to $500 \mathrm{GHz}$. Evaluating the different modes of the combs allows us to quantify the dynamic properties of this two-layer system with great precision. Deviations of the frequencies of higher modes from a linear relation can be quantitatively understood. The time domain traces show clearly defined pulses which are detected in regular time intervals after each roundtrip in the acoustic cavity formed by the membrane and the metal film. By analyzing the individual reflected pulses and their evolution in time, damping times for the whole frequency range are determined. We analytically derive a deviation of the individual comb modes from integer values of the fundamental frequency which is corroborated by the experiments.
\end{abstract}

DOI: 10.1103/PhysRevB.88.205202

PACS number(s): 68.60.Bs, 62.80.+f, 43.35.+d, 43.38.+n

The dynamics of high-frequency acoustic waves in semiconductors are of great interest for both fundamental and applied science. ${ }^{1-3}$ Even for intensively studied and technologically most important semiconductors like $\mathrm{Si}$, the properties of high frequency $(\mathrm{GHz}-\mathrm{THz})$ acoustic phonons are not fully understood. ${ }^{4-6}$ At the same time, heat transfer, which is linked to acoustic wave propagation, is of current interest to basic science as well as for the performance limits of semiconductor devices. ${ }^{7}$ Especially the regime where coherent acoustic phonons are in the frequency range relevant for heat transfer, e.g., of thermal phonons, is interesting. ${ }^{8}$ Furthermore, phonon lifetimes, limited by intrinsic and extrinsic scattering processes, are not well known for most materials. ${ }^{9,10}$

Frequency combs are important tools for high precision metrology and are common in the field of optics. An optical frequency comb nowadays can be generated from a modulated femtosecond laser which generates a spectrally broad comb with a mode spacing given by the inverse of the cavity roundtrip time. ${ }^{11}$ Phononic microcavities are a promising approach to understanding the acoustic behavior in multilayer systems. ${ }^{12,13}$ But there are only a few examples of frequency combs related to mechanical excitation of microstructures and semiconductors in the literature and none of them are in the high gigahertz frequency range. ${ }^{14-16}$ Recently, standing acoustic modes in the high $\mathrm{GHz}$ range were generated in silicon (Si) membranes, although only odd modes or the fundamental mode $^{10}$ could be efficiently excited. ${ }^{6}$ Also optical phonon modes in $\mathrm{Si}$ were excited at $\mathrm{THz}$ frequencies and ascribed as phononic frequency comb. ${ }^{17}$

In this article we demonstrate the generation and detection of acoustic combs in the $100-\mathrm{GHz}$ frequency range. We use femtosecond pump-probe spectroscopy to access acoustic dynamics in a thin aluminum (Al)/Si-layer system over a wide range of frequencies in a single measurement. We excite frequency combs which consist of 11 up to 45 modes in a frequency range from 10 to $500 \mathrm{GHz}$. The analysis of the comb allows us to investigate the dynamic properties induced in the two-layer system and to determine the frequency-dependent damping. By measuring on membranes with Al layers with different thicknesses we gain significant insight into the optical impulsive generation and detection of the acoustic strain in the $\mathrm{Al} / \mathrm{Si}$-layer system. A simple model is used to simulate the system taking into account strain generation by thermoelastic expansion (TE) as well as by a deformation potential (DP). We demonstrate excellent agreement between experiments and simulations. This paper is organized as follows: First, a sample description is given, second, the pump-probe setup is introduced, third, the experimental results are presented leading to a spectral domain analysis including intrinsic two-layer properties, and at last, the phonon lifetimes for the different samples are calculated.

\section{SAMPLE DESCRIPTION}

All investigated samples have the same layer structure with varying thicknesses. The sample consists of a thin $\mathrm{Al}$ film on top of a single crystalline Si membrane (Fig. 1). The membrane is obtained from a commercially available $\mathrm{Si}$-on-insulator wafer, ${ }^{18}$ which is wet etched from the backside with potassium hydroxide using a Si-nitride etch mask. The oxide layer which acts as an etch stop is removed by consecutive dipping of the wafer in hydrofluoric acid. The free-standing Si membranes are $600 \times 600 \mu \mathrm{m}^{2}$ large with thicknesses ranging from 330 to $360 \mathrm{~nm}$. One membrane is reactive-ion etched with sulfur hexafluoride and oxygen, resulting in a $d_{\mathrm{Si}}=328 \pm 2-\mathrm{nm}-$ thick membrane, while the other three membranes are not etched, having thicknesses of $350 \pm 2 \mathrm{~nm}$. On top of the etched membrane, a $d_{\mathrm{Al}}=17 \pm 1$-nm-thick Al film is evaporated, while on the other three membranes $\mathrm{Al}$ is sputtered with thicknesses of $10 \pm 1,23 \pm 1$, and $40 \pm 4 \mathrm{~nm}$, respectively. The experimental results show, as will be discussed later, that the general properties of the system is not influenced by the etching. The layer thicknesses were determined by atomic force microscopy and ellipsometry. 


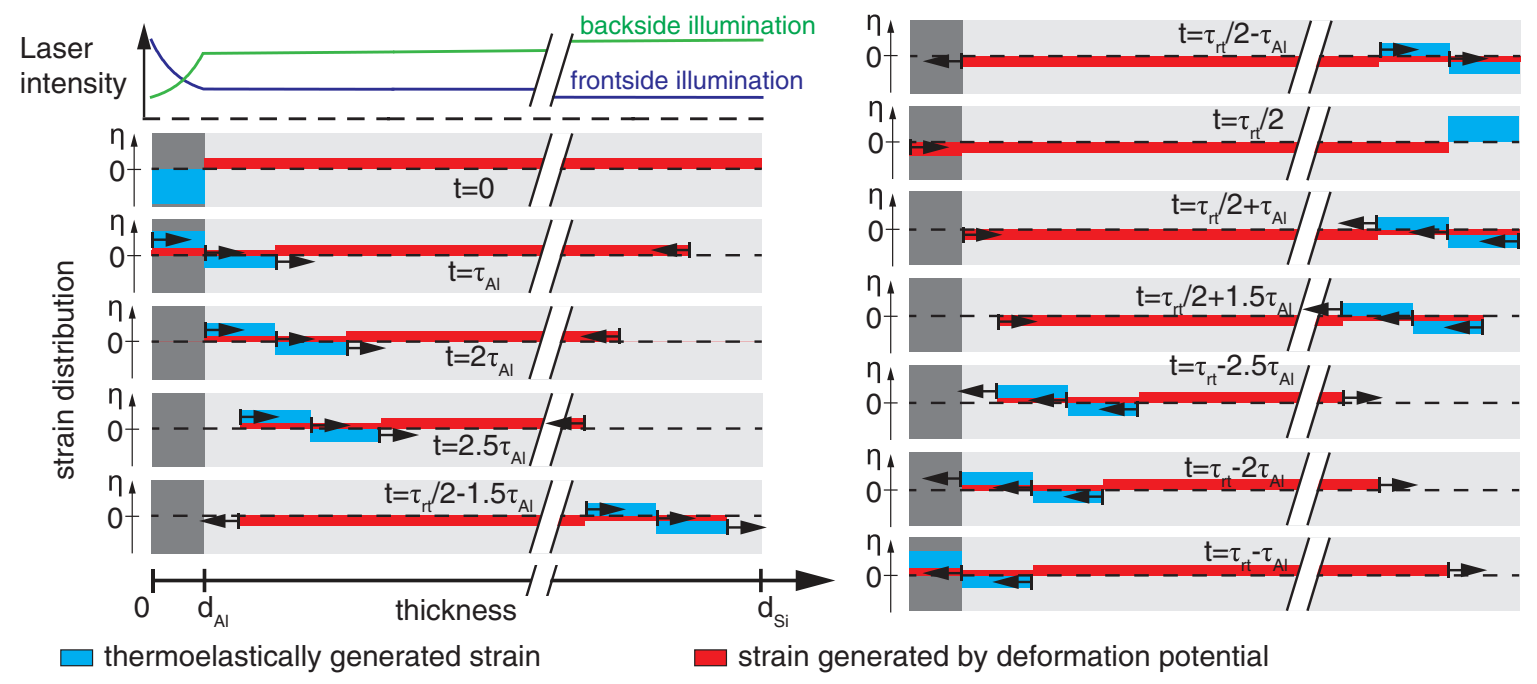

FIG. 1. (Color online) Illustration of the laser intensity and strain distribution and evolution in the sample. On the top left the schematic intensity of the penetrating laser pulse is plotted for frontside and backside illumination. Beneath at time $t=0$ the generated acoustic strain by thermoelastic excitation in the $\mathrm{Al}$ (blue) and by deformation potential in the $\mathrm{Si}$ (red) is plotted. Below and on the right the consecutive time evolution for relevant strain distributions is plotted. The arrows indicate the propagation direction of the various strain fronts. $\tau_{\mathrm{rt}}$ and $\tau_{\mathrm{Al}}$ are the roundtrip time for an acoustic phonon in the sample and the time for an acoustic phonon to pass the aluminum film once, respectively.

\section{PUMP-PROBE SETUP}

The acoustic dynamics of the membrane were investigated using high-speed asynchronous optical sampling (ASOPS). ${ }^{19}$ ASOPS is a pump-probe technique utilizing two coupled femtosecond Ti:sapphire oscillators with a repetition rate of $f_{R}=800 \mathrm{MHz}$ with pulse lengths of nominally $50 \mathrm{fs}$, defining a measurement window given by $1 / f_{R}$ of $1.25 \mathrm{~ns}$. The oscillators have an actively stabilized fixed offset in the repetition rate of $5 \mathrm{kHz}$. Thereby the pump and probe pulses have a linear increasing time delay. Due to the high repetition rate, it is possible to accumulate a large number of measurements, resulting in a signal-to-noise ratio better than $10^{7}$. The pump laser power used to excite the membrane is $20 \mathrm{~mW}$ at a wavelength of $790 \mathrm{~nm}$ and the probe laser power is $3.5 \mathrm{~mW}$ at a wavelength of $820 \mathrm{~nm}$. Pump and probe pulses are focused on the Al side of the sample using a $50 \times$ NIR microscope objective (Mitutoyo) with a NA of 0.42 . The spatial FWHM of the pump and probe spot is below $2 \mu \mathrm{m}$. The thickest sample is also probed from the backside, i.e., the $\mathrm{Si}$ side. The generation and detection mechanism of the acoustic dynamics of the membranes is strongly depending on the thickness of the Al. For thick Al films the generation is dominated by $\mathrm{TE}$ in the $\mathrm{Al}$ layer, while for the thinner $\mathrm{Al}$ films the excitation by DP in the silicon membrane becomes more prominent. For thick $\mathrm{Al}$ layers the detection process is governed by the photoelastic effect in the Al layer, while for thinner layers a dynamic Fabry-Perot-like effect, i.e., the thickness variation of the $\mathrm{Si} / \mathrm{Al}$ system, becomes dominant. This will be explained in detail in the following sections which are divided according to the aforementioned effects.

\section{EXPERIMENTAL RESULTS}

\section{A. Thermoelastic excitation in thick Al layer}

First the membrane with the thickest Al film $(d=40 \mathrm{~nm})$ will be discussed, where the influence of the deformation potential can be neglected because $99 \%$ of the pump light is absorbed in the 40 -nm-thick Al layer. ${ }^{20}$ Thereby almost no light is absorbed in the Si layer. Figure 2 shows the extracted time trace of the measured signal without the electronic background. The trace displays repeating equidistant pulses appearing first at $98 \mathrm{ps}$ and consecutive multiples of that time. Each pulse has a characteristic shape consisting of two maxima and a minimum in between. A close up of the first pulse is shown in the inset (the minimum amplitude is normalized to -2 ). The pulse decays exponentially over the time window and gains additional oscillatory components at its sides.

The signal is a convolution of the TE strain generated in the $\mathrm{Al}$ layer ${ }^{21,22}$ and the photoelastic detection in the $\mathrm{Al}^{21}$ (see schematic in Fig. 1). The three consecutive phases of the detected dominant acoustic pulse (see inset in Fig. 2) can be identified with the detection of three separate strain fronts.

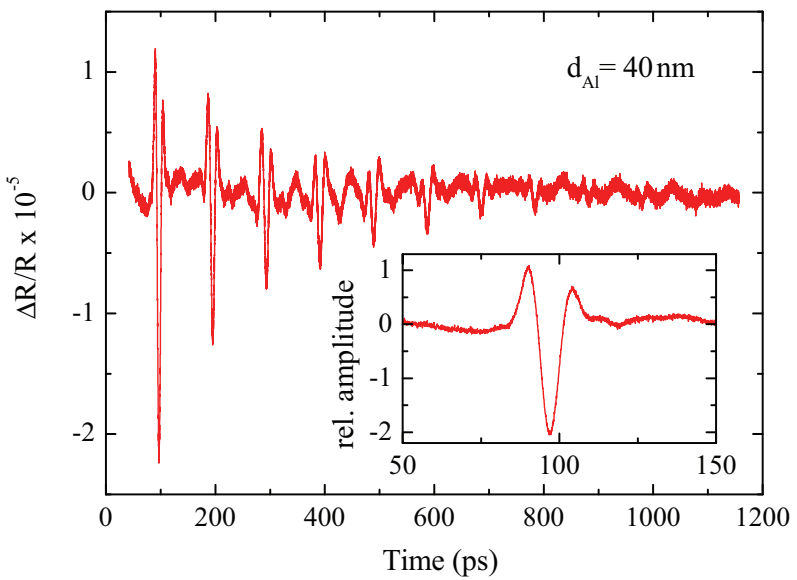

FIG. 2. (Color online) Time-resolved modulation of the reflected probe beam $\Delta R / R_{0}$ without electronic background. A close up of the first pulse normalized to an amplitude of -2 is shown in the inset. $350 \mathrm{~nm}$ membrane with $40 \mathrm{~nm} \mathrm{Al}$ on top. 
The first of them is generated at the $\mathrm{Al} / \mathrm{Si}$ interface by TE and is emitted straightforward into the Si membrane, the second one is generated at the free surface of $\mathrm{Al}$ by TE as well and enters the $\mathrm{Si}$ with a time delay of $\tau_{\mathrm{Al}}=d_{\mathrm{Al}} / v_{\mathrm{Al}}$, where $v_{\mathrm{Al}}$ is the longitudinal speed of sound in $\mathrm{Al}$, relative to the first one, and the third, generated similarly to the first one at the $\mathrm{Al} / \mathrm{Si}$ interface but counterpropagating, consequently is emitted into the $\mathrm{Si}$ only after the reflection at the free surface of $\mathrm{Al}$ with an additional delay of $2 \tau_{\mathrm{Al}}$ relative to the first strain front. The build-up and the evolution in time of these fronts after the impulsive strain generation at $t_{0}$ is schematically shown in Fig. 1 (blue strain). As can be seen the fronts produce a bipolar strain pulse in the system. When this bipolar pulse is detected photoelastically in the Al layer, due to the short penetration depth of light, then - qualitatively speaking — the derivative of the strain is detected, giving rise to a tripolar pulse in the time domain.

The relative strain of these emitted fronts can be normalized to the first front (half the TE strain) and are therefore on the order of $1,-2$, and $1-\epsilon$ for the first, second, and third front, respectively. This corresponds to the two main maxima and the minimum of the pulse in the inset of Fig. 2. The factor $\epsilon$ takes into account the role of heat conduction between Al layer and $\mathrm{Si}$, leading to the asymmetry between the first and the third front. ${ }^{23}$ Due to the reflection of $7 \%{ }^{24}$ at the $\mathrm{Si} / \mathrm{Al}$ interface each pulse gets additional oscillatory components at the front and back of the pulse, which is clearly visible in the time trace for the pulse. The time between two pulses matches precisely the roundtrip time of the longitudinal acoustic phonon of the $\mathrm{Al} / \mathrm{Si}$ layer system. The system is an acoustic cavity in which the $\mathrm{Al}$ /air top surface and the $\mathrm{Si} /$ air bottom surface act as acoustic mirrors forming the cavity. The roundtrip time is given by the simple relation $2\left(\tau_{\mathrm{Al}}+\tau_{\mathrm{Si}}\right)$, with $\tau_{\mathrm{Si}}=d_{\mathrm{Si}} / v_{\mathrm{Si}}$, where $v_{\mathrm{Si}}$ is the longitudinal speed of sound in Si. Making use of the measured values for the $\mathrm{Al}$ and $\mathrm{Si}$ thickness, and a speed of sound for $\mathrm{Si}$ of $8430 \mathrm{~m} / \mathrm{s},{ }^{25}$ and for $\mathrm{Al}$ of $6320 \mathrm{~m} / \mathrm{s},{ }^{26}$ we obtain a roundtrip time of $97.6 \mathrm{ps}$, in excellent agreement with the experimentally observed value.

\section{B. Excitation through deformation potential for thin Al layers}

After analyzing the membrane with the thick aluminum film in the previous section, membranes with thinner Al films will be discussed next. In this case the pump light is only partially absorbed in the $\mathrm{Al}$ and a part is also absorbed in the $\mathrm{Si}$. This generates an additional strain by the DP (about one magnitude larger than TE). ${ }^{5,27,28}$ Because the samples are similar in their physical behavior, both samples will be discussed simultaneously. In the sample with a 17-nm-thick Al film $89 \%$ (in the 23-nm-thick Al film 95\%) of the pump light is absorbed in the Al. Figures 3 and 4 show the extracted time traces of the measured signal without the electronic background for the 17 and the $23 \mathrm{~nm} \mathrm{Al}$ sample, respectively. The trace displays two sorts of repeating equidistant pulses: a smaller one appearing first at $41.5 \mathrm{ps}$ (45.5 ps) (with the shape of a single minimum), and a larger one appearing first at $83.0 \mathrm{ps}$ (91.1 ps). A close up of the later is shown in the inset (the minimum amplitude is normalized to -2 ). Both pulses decay exponentially over the entire time window and acquire new oscillatory components on their sides for longer time delays.

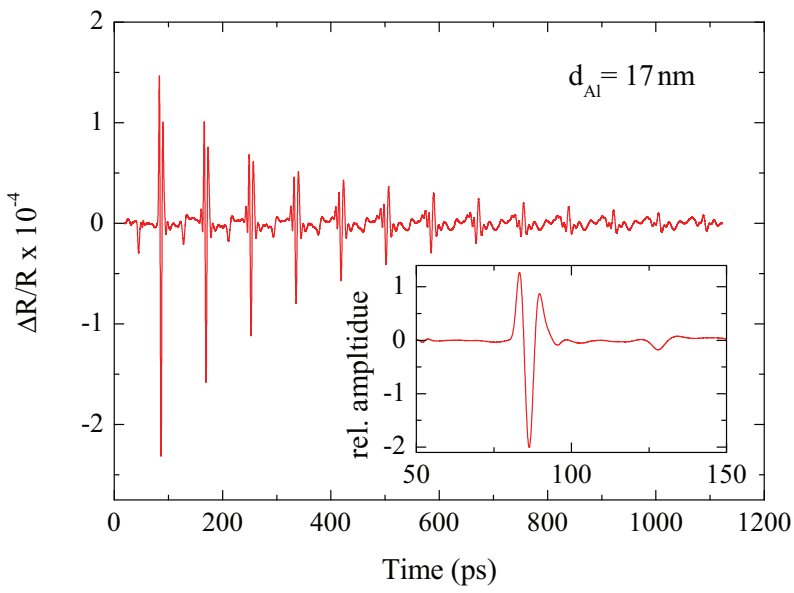

FIG. 3. (Color online) Time-resolved modulation of the reflected probe beam $\Delta R / R_{0}$ without electronic background of the $328 \mathrm{~nm}$ Si membrane with $17 \mathrm{~nm} \mathrm{Al}$ on top. A close up of the large pulse normalized to an amplitude of -2 is shown in the inset.

The pulse detected before the first arrival of the large pulse at the time $41.5 \mathrm{ps}$ (45.5 ps) and later in the middle between two successive large pulses (see Figs. 3 and 4) corresponds to the detection of strain fronts, which are generated on the back surface of the Si membrane entirely by the DP mechanism (relative amplitude of $\mu$ ). Similarly to the previous case of the thermoelastic generation in $\mathrm{Al}$, the strain fronts propagate in front and back direction [see Fig. 1 blue (TE) and red (DP) strain]. After half the roundtrip time the fronts generated by DP reach the Al layer, where they are photoelastically detected, thereby creating the dip. The roundtrip time is shorter than in the $40 \mathrm{~nm}$ sample, matching the new cavity length. The additional pulses are not the only visible difference in the time transient compared to the sample with the thicker Al layer. The strain generated by the DP is also apparent on the large pulses. It influences the two maxima of the pulses, changing their amplitudes [see Fig. 1 superpositioned strain by DP (red)]. The relative magnitudes

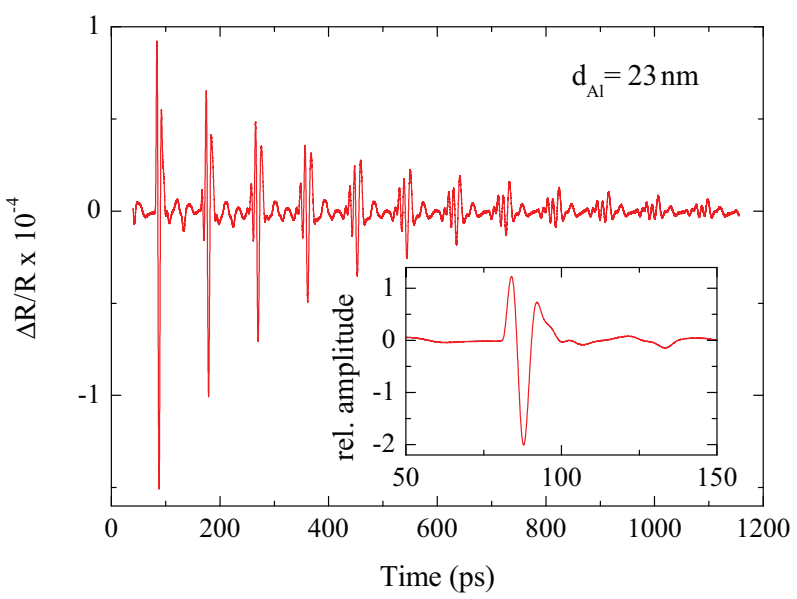

FIG. 4. (Color online) Time-resolved modulation of the reflected probe beam $\Delta R / R_{0}$ without electronic background of the $350 \mathrm{~nm}$ Si membrane with $23 \mathrm{~nm} \mathrm{Al}$ on top. A close up of the large pulse normalized to an amplitude of -2 is shown in the inset. 
of the three phases of the detected pulses become $(1+\mu)$, (-2), $(1+\mu-\epsilon)$, respectively (see inset in Figs. 3 and 4). The factor $\mu$ quantifies the influence of the DP strain, leading to a relative amplitude for the small pulse proportional to the large pulse of $-2 \mu$. The comparison of the amplitudes of the consecutive small and large pulses show that for the $17 \mathrm{~nm}$ Al layer sample $\mu \approx 0.26$ (for the $23 \mathrm{~nm}$ Al layer sample $\mu \approx 0.21)$. As will be discussed later, the DP effect has a pronounced effect on the frequency-domain spectra.

\section{Detection by dynamic Fabry-Perot interference}

In the previous sections only the photoelastic effect in the $\mathrm{Al}$ layer was important for detection, because of probing at this side. Under certain conditions also Fabry-Perot interference can become important: The first example for pumping and probing at the backside is the sample with a $40 \mathrm{~nm} \mathrm{Al}$ layer discussed previously for frontside probing. Instead of illuminating the sample from the Al covered frontside (no light reaching the silicon), it is illuminated from the backside. Thereby around 4\% intensity is absorbed in the Si before the light reaches the $\mathrm{Al}$ film and is fully absorbed (see schematic in Fig. 1). In this way additional strain is generated by DP. Figure 5 shows the extracted time trace of the measured signal without the electronic background. Like in the 17 and $23 \mathrm{~nm}$ Al layer samples both large and small pulses are visible, the small one appearing first at 50 ps and the large one at $100 \mathrm{ps,}$ respectively. The large one at 100 ps has the same features as in the measurement from the frontside, but the relative amplitudes $(1+\mu,-2,1+\mu-\epsilon)$ differ (see inset in Fig. 5). The small pulse at half the roundtrip time is comparable in relative amplitude $(-2 \mu)$ to the minimum of the large pulse (rel. amplitude of -2 ). Analyzing both pulses the value of $\mu$ can be evaluated to be around 0.9. The large pulse seems to diminish equally fast as in the frontside measurement but a slow oscillation is visible throughout the entire time window. This is caused by the slower damping of low-frequency modes compared to higher frequencies as well as a difference in the detection process.

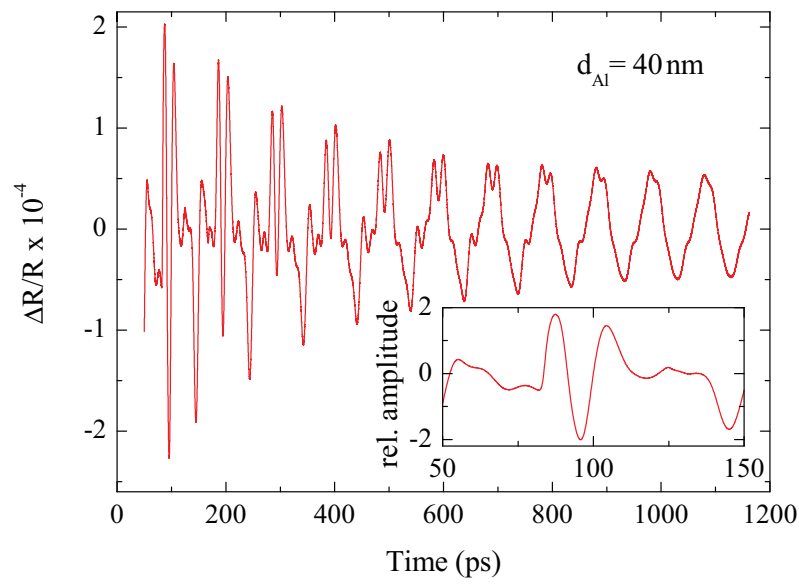

FIG. 5. (Color online) Time-resolved modulation of the reflected probe beam $\Delta R / R_{0}$ without electronic background of the $350 \mathrm{~nm}$ $\mathrm{Si}$ membrane with $40 \mathrm{~nm} \mathrm{Al}$ on top measured from the backside. A close up of the first pulse normalized to an amplitude of -2 is shown in the inset.
Comparing to previous results on bare $\mathrm{Si}$ membranes it was shown that in $\mathrm{Si}$ the photoelastic contribution gets very small compared to the contribution from the change in the optical cavity thickness (here called Fabry-Perot effect). ${ }^{5}$ The two end surfaces of the membrane act as mirrors of a cavity, building a Fabry-Perot cavity. The moving strain and the corresponding displacements of the end surfaces induce constructive and destructive optical interference, hence the reflectivity is changed.

In the $\mathrm{Al}$ covered membranes discussed in this article there is also a Fabry-Perot-like effect due to surfaces, the air-Si interface and the $\mathrm{Si}-\mathrm{Al}$ interface, moving relative to each other. The strain fronts in the Si (front- and backside) still travel back and forth in the membrane [see Fig. 1 (red)]. Because the interferometric detection is sensitive to the displacements, which are the integrals of strain, the slow oscillation is visible over the whole time transient. In contrast, the TE strain [see Fig. 1 (blue)] is bipolar, so it can only be detected at the surfaces (at the Al surface the PE detection is still present), where the integral becomes nonzero.

To suppress the contribution from the photoelastic detection in favor of the Fabry-Perot detection a membrane with a $10 \mathrm{~nm} \mathrm{Al} \mathrm{film} \mathrm{on} \mathrm{top} \mathrm{was} \mathrm{measured} \mathrm{from} \mathrm{the} \mathrm{frontside.}$ The Al absorbs $74 \%$ of the pump light, so probe light from the backside of the $\mathrm{Si}$ is still able to be measured. Due to the small thickness of the film, corresponding to a short absorption distance, it was also possible to generate a very short acoustic strain pulse. Figure 6 shows the extracted time trace of the measured signal without the electronic background. In the beginning of the transient alternating maxima (first at $41.5 \mathrm{ps}$ ) and minima (first at $83.0 \mathrm{ps}$ ) can be seen, while at later times only a slow oscillation is visible. The peaks appear at every half integer of the roundtrip time. The strong peaks in the beginning are caused by strain excited through TE, but differ from the ones shown previously. Instead of a pulse shape with three extremes (maximum/minimum/maximum), pulses at odd half roundtrip times show a single maximum and pulses for even half roundtrip times show a single minimum. These peaks are caused by the change in thickness induced by the strain excited in the $\mathrm{Al}$, when it reaches the different surfaces.

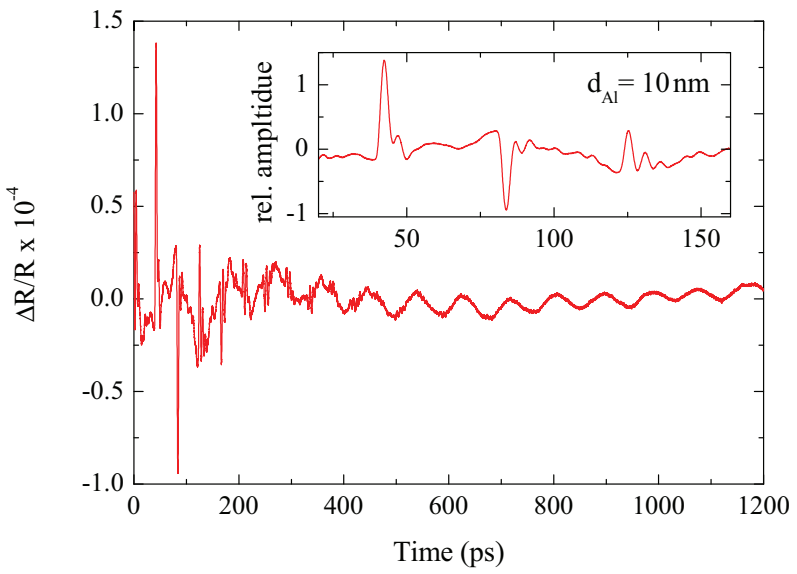

FIG. 6. (Color online) Time-resolved modulation of the reflected probe beam $\Delta R / R_{0}$ without electronic background of the $328 \mathrm{~nm} \mathrm{Si}$ membrane with $10 \mathrm{~nm} \mathrm{Al}$ on top. A close up is shown in the inset. 


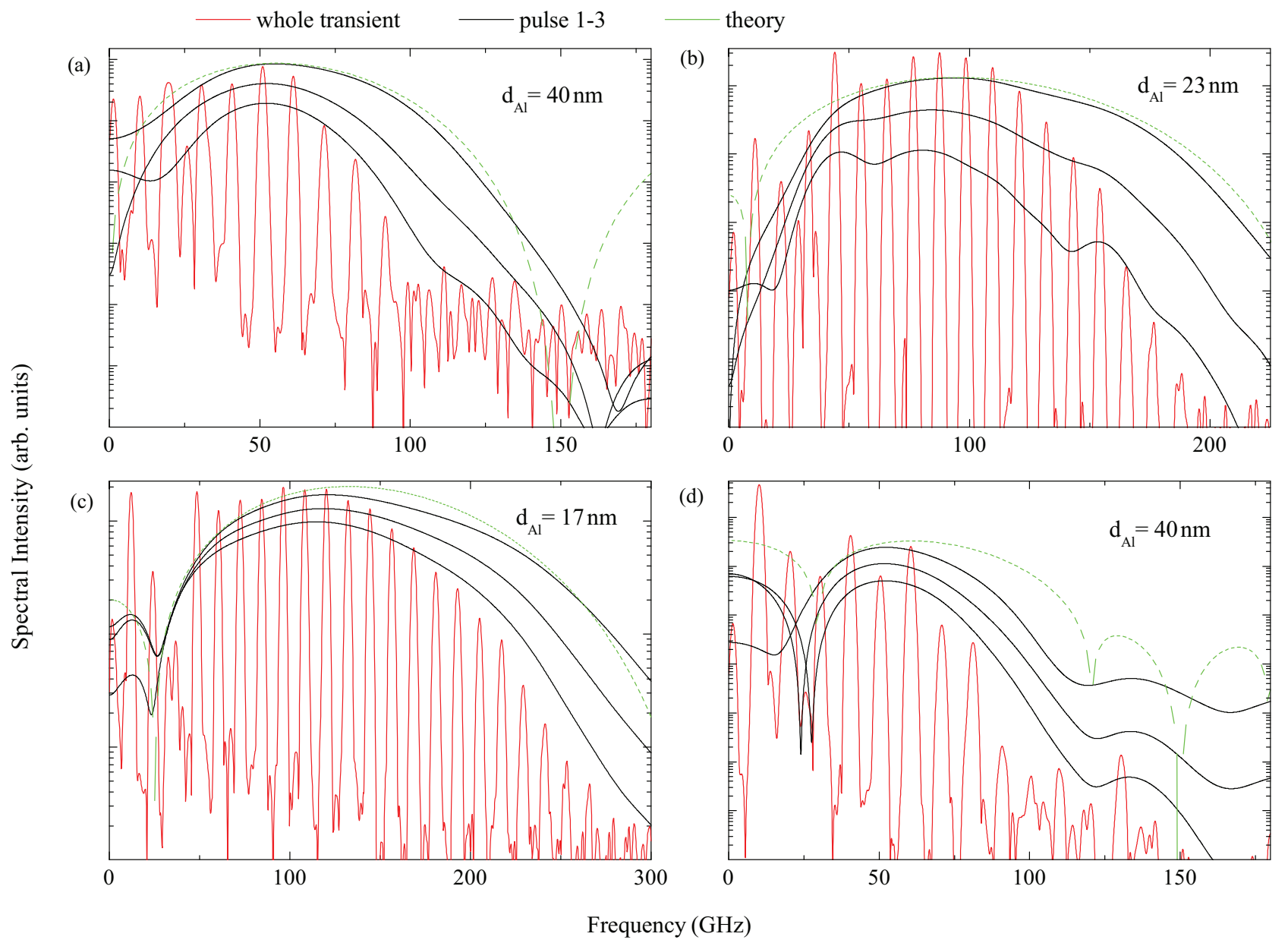

FIG. 7. (Color online) Fourier spectra of the whole time transient (red) and the first three pulses of the (a) 40-nm-thick Al sample, (b) 17-nm-thick sample, (c) $23 \mathrm{~nm}$ sample, and (d) backside measurement of the $40 \mathrm{~nm}$ sample. The dashed line shows the theoretical fit of the excited pulse.

When the bipolar TE strain pulse moves inside the $\mathrm{Si}$, the pulse is localized in the membrane and the displacement of the surfaces, given by the integral over the strain, is zero. We detect the pulses when they are coming to the surface, the integral over the strain becomes nonzero and the surface starts to displace. This mechanism also explains the unipolar shape of the detected peaks. Due to the phase shift of $\pi$ at the reflection of the acoustic pulse, the maximum strain at the $\mathrm{Al}$ and $\mathrm{Si}$ surface has a different sign and the surface moves in a different direction at the $\mathrm{Al}$ and $\mathrm{Si}$ surface (see Fig. 1), leading to the alternating change in optical reflectivity. The maxima correspond to the strain reaching the Si-air interface and the minima correspond to strain reaching the $\mathrm{Si}$-Al-air interface. Due to the larger speed of sound in Si than in Al the maxima are shorter in time than the minima by a factor of $4 / 3$ given by the ratio between $v_{\mathrm{Si}}$ and $v_{\mathrm{Al}}$.

To get a better insight of the phonon dynamics in this system, further analysis in the spectral domain is performed.

\section{FREQUENCY DOMAIN ANALYSIS}

In the spectral domain the measured pulse trains correspond to frequency combs, which can be clearly observed in Figs. 7(a)-7(d) (corresponding to $d_{\mathrm{Al}}$ of $40,23,17 \mathrm{~nm}$, and backside measurement of the $d_{\mathrm{Al}}=40 \mathrm{~nm}$ sample) and Fig. 8 $\left(d_{\mathrm{Al}}=10 \mathrm{~nm}\right.$ sample), where the fast Fourier transforms (FFT) of the whole extracted time transient of the samples

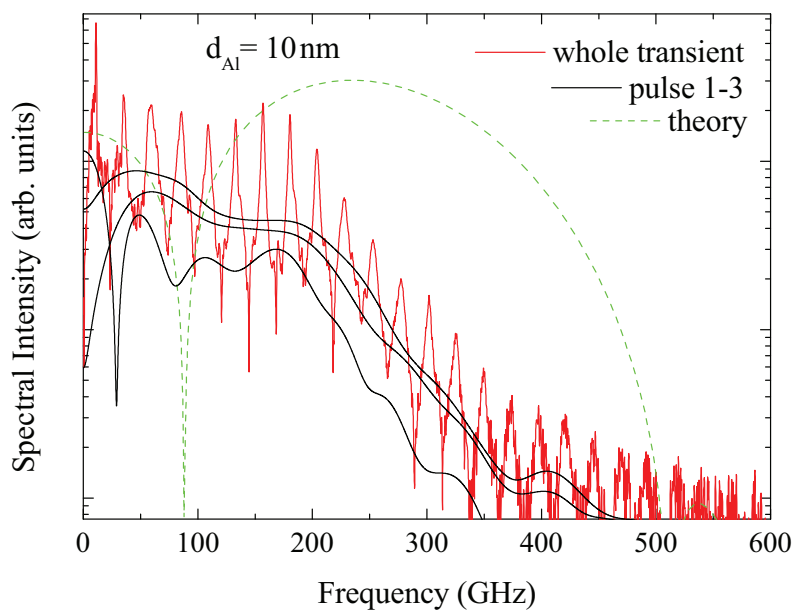

FIG. 8. (Color online) Fourier spectrum of the whole time transient (red) and the first three pulses of the $10 \mathrm{~nm}$ Al layer sample. The dashed line shows the expected Fourier spectrum of the excited pulse. Note the change of the frequency scale compared to Fig. 7. 
(displayed in Figs. 2 to 5) are shown (red curve). The comb is characterized by nearly regularly spaced narrow peaks, which are observed to frequencies up to several $100 \mathrm{GHz}(200,300$, and $500 \mathrm{GHz}$, for decreasing $d_{\mathrm{Al}}$, respectively). The observed peaks correspond to the vibrational eigenmodes of the thin bilayered $\mathrm{Al} / \mathrm{Si}$ membrane, and are a direct consequence of the boundary conditions at the top Al/air and the bottom Si/air free surfaces that act as two high-quality acoustic reflectors, which constitute the acoustic cavity that confines the longitudinal acoustic strain. ${ }^{5,6}$ The ground mode of the system is found at $f_{0}=10.0 \mathrm{GHz}(11.0,12.05$, and $12.05 \mathrm{GHz})$, and the even and odd higher harmonics are visible up to the 9th (18th, 24th, and 43rd) order. The spectral intensity of the comb's envelope spreads over a wide range of frequencies, presenting a maximum around $50 \mathrm{GHz}$ (100, 120, and 300 $\mathrm{GHz}$ ). For the thin sample, only the odd modes are visible due to the suppressed photoelastic and enhanced Fabry-Perot detection.

Due to the fact that the pulses are well separated and distinguished in the time domain (Figs. 2-6), it is possible to analyze their individual spectral behavior. This has been accomplished by extracting and transforming the individual pulses from the time trace by centering each of them in a time window corresponding to one roundtrip [i.e., 100 ps $(91.1,83$, half roundtrip $41.5 \mathrm{ps}$ )]. The result of the FFTs of the first three isolated pulses is shown superimposed in Figs. 7(a)7(d) and 8. As can be seen, the resulting general spectral behavior is as expected similar to the frequency comb's envelope.

Noticeable in the spectra of the samples with $17 \mathrm{~nm} \mathrm{Al}$ layer is a strong dip at $\sim 23 \mathrm{GHz}$, which is also visible in the comb's envelope. Additionally, from the comparison of the three spectra of the consecutive individual pulses, a faster decay in intensity for higher frequencies is evident. The dip appearing in the comb's spectrum at $\sim 23 \mathrm{GHz}$ can be explained as the result of the competition between the two involved strain generation mechanisms: DP and TE. The DP in the Si is the dominant contribution to the spectral weight of the induced stress at low frequencies. The TE in the Al is the dominant contribution to the spectral weight at higher frequencies. Since the effect of DP and TE on the strain generation have opposite signs, ${ }^{29}$ a spectral analysis demonstrates that under the condition $0<\mu \ll 1$ there should be a complete mutual compensation of the two mechanisms at a certain frequency $f_{\mu} \approx(\sqrt{2 \mu} / \pi) f_{\mathrm{Al}} \ll f_{\mathrm{Al}}$, where $f_{\mathrm{Al}}=1 /\left(2 \tau_{\mathrm{Al}}\right)$ is the fundamental resonance frequency of the free-standing Al film. This also provides an estimate for the frequency at which the spectrum has an absolute maximum, which is in our case around $175 \mathrm{GHz}(50 \mathrm{GHz}$ for the $40 \mathrm{~nm}$ sample, $130 \mathrm{GHz}$ for the $23 \mathrm{~nm}$ sample, and $300 \mathrm{GHz}$ for the $10 \mathrm{~nm}$ sample), corresponding very well with the experimental results.

Figures 7(a)-7(d) (dashed line) and 8 show the corresponding Fourier spectra of the dominant pulse calculated with the strain profile generated by TE and DP shown in Fig. 1, including reverberation at the $\mathrm{Al} / \mathrm{Si}$ interface using the following values: speed of sound for $\mathrm{Si}$ along the [100] direction (value of bulk silicon): $v_{\mathrm{Si}}=$ $8430 \mathrm{~m} / \mathrm{s}$, speed of sound for the thin Al film: $v_{\mathrm{Al}}=$ $6000 \mathrm{~m} / \mathrm{s}$, the thicknesses of the various samples are given in
TABLE I. Fit parameters for the different samples.

\begin{tabular}{lcrrr}
\hline \hline$v_{\mathrm{Al}}(\mathrm{m} / \mathrm{s})$ & $Z_{\mathrm{Al}}(\mathrm{GPa} \mathrm{s} / \mathrm{m})$ & $d_{\mathrm{Al}}(\mathrm{nm})$ & $d_{\mathrm{Si}}(\mathrm{nm})$ & $\mu$ \\
\hline 6000 & 17.0 & 10 & 336 & 0.33 \\
& & 17 & 326 & 0.055 \\
$v_{\mathrm{Si}}(\mathrm{m} / \mathrm{s})$ & $Z_{\mathrm{Si}}(\mathrm{GPa} \mathrm{s} / \mathrm{m})$ & 23.5 & 351 & 0.01 \\
8430 & 19.6 & 42 & 358 & $0 / 1$ \\
\hline \hline
\end{tabular}

Table I. The spectral amplitude is given by

$$
\begin{aligned}
\tilde{D}(\omega)= & \tilde{D}_{\mathrm{TE}}+\tilde{D}_{\mathrm{DP}} \propto 2 \frac{\sin ^{4}\left(\omega \tau_{\mathrm{Al}} / 2\right)}{\left(\omega \tau_{\mathrm{Al}} / 2\right)^{2}} \\
& -\bar{\mu} \frac{\sin ^{2}\left(\omega \tau_{\mathrm{Al}} / 2\right)}{\left(\omega \tau_{\mathrm{Al}} / 2\right)^{2}} \cos \left(\omega \tau_{\mathrm{Al}}\right),
\end{aligned}
$$

where the first term of the sum $\left(\tilde{D}_{\mathrm{TE}}\right)$ denotes the contribution of the TE strain and the second term $\left(\tilde{D}_{\mathrm{DP}}\right)$ of the strain generated by DP. The measured spectra can be reproduced very well. For the samples, where the Fabry-Perot detection mechanism is dominant (see Fig. 8), the frequency comb does not fit as well to the FFT of the experimentally obtained single pulses due to the symmetric detection.

The experimental observation of a dip instead of the expected absolute zero is mostly due to the influence of the thermal conduction to the part of the signal which is generated in the Al. The heat conduction increases the low-frequency part of the TE generated signal and hinders the complete compensation of the DP and TE contributions.

The influence of the DP can be changed by probing from the backside. Figure 7(d) shows the FFT of the backside measurement of the sample with $40 \mathrm{~nm} \mathrm{Al}$. The spectrum looks very similar to the frontside spectrum, but the first peak is more intense compared to the rest of the spectrum. Also a peak at $130 \mathrm{GHz}$, which was suppressed in the frontside spectrum, appears. The FFTs of the first three pulses are shown in black with a pronounced dip at $25 \mathrm{GHz}$, corresponding well to our theoretical model. The dip is not well visible in the whole transient spectra. We attribute this to the thick film and the high absorption and correspondingly to a larger effect of the thermal conductance.

\section{DISTINCTIVE TWO-LAYER MODES}

A careful analysis of the frequency of the individual peaks of the combs show that they do not match exactly integer multiples of the ground mode $f_{0}$, as previously observed for the case of bare freestanding Si membranes. In Fig. 9 the normalized deviation of the individual peaks with frequency $f_{n}$ from the linear expression $n \cdot f_{0}$ is plotted for all the membranes with different $\mathrm{Al}$ thicknesses. $n$ is the integer indicating the mode number. Note that the ground mode's frequency matches the inverse of the roundtrip time of the system. The plot shows that the mode's frequency oscillates around zero, which defines the linear dispersion, with maximal deviations on the order of $3 \%$. In order to understand this systematic oscillatory behavior for a two-layer system, calculations were performed solving the equation of motion for the displacement field of longitudinal acoustic vibrations for the free-standing two-layer Al/Si system along 


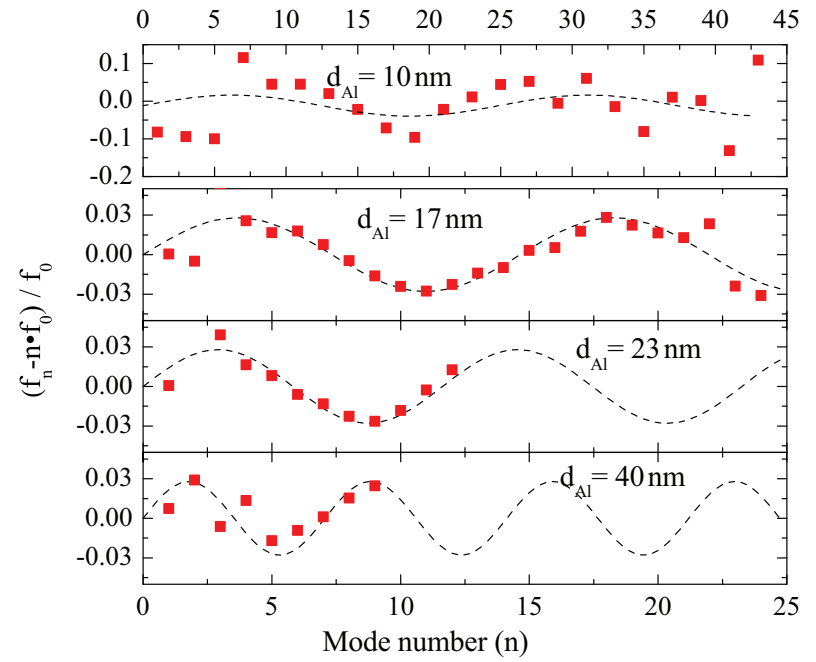

FIG. 9. (Color online) Deviation of the excited mode frequencies $f_{n}$ from the linear dependence given by the ground mode times mode number $\left(n \cdot f_{0}\right)$ divided by ground mode frequency. The dashed line corresponds to the theoretical values.

the confinement direction, and obtaining the eigenfrequencies. Both layers are assumed to be perfectly bonded to each other. The possible eigenmodes $\omega$ of the system are given by the two-layer equation:

$$
Z_{\mathrm{Si}} \tan \left(\omega \tau_{\mathrm{Si}}\right)+Z_{\mathrm{Al}} \tan \left(\omega \tau_{\mathrm{Al}}\right)=0 .
$$

As first approximation we assume similar impedances $Z_{\mathrm{Si}} \approx$ $Z_{\mathrm{Al}}$, so that the equation can be simplified: $\sin \left[\omega\left(\tau_{\mathrm{Si}}+\tau_{\mathrm{Al}}\right)\right]=$ 0 . The solutions are rather simple and given by $\omega_{n}\left(\tau_{\mathrm{Si}}+\tau_{\mathrm{Al}}\right)=$ $\pi n$ with

$$
\omega_{n}=\frac{\pi}{\tau_{\mathrm{Si}}+\tau_{\mathrm{Al}}} n=: \omega_{1} n,
$$

$n$ being a positive integer. $\omega_{1} / 2 \pi$ corresponds to the inverse roundtrip time in the two-layer system of the longitudinal acoustic phonon.

In the next approximation we look for small perturbations of the frequency $\omega=\omega_{n}+\Delta \omega$ induced by the difference in impedances. We obtain for the modulation:

$$
\frac{\Delta \omega}{\omega_{1}}=\frac{\Delta f}{f_{1}}=\frac{1}{2 \pi}\left[1-\frac{Z_{\mathrm{Al}}}{Z_{\mathrm{Si}}}\right] \sin \left(\frac{2 \pi}{1+\tau_{\mathrm{Si}} / \tau_{\mathrm{Al}}} n\right) .
$$

Equation (3) is plotted in Fig. 9 (dashed line), where the best agreement to the experimental data was achieved using the values given in Table I, while the impedances of $\mathrm{Si}$ and $\mathrm{Al}$ were taken to be $Z_{\mathrm{Si}}=19.6 \mathrm{GPa} \mathrm{s} / \mathrm{m}$ and $Z_{\mathrm{Al}}=16.2 \mathrm{GPa} \mathrm{s} / \mathrm{m}$, respectively. Note that both the period and the amplitude of the oscillation are matched. Only the $10 \mathrm{~nm}$ sample shows-despite the large number of contributing modes-a comparably strong deviation. The parameters were chosen for a good agreement to the experimental data and are very close to the tolerances of the values determined by ellipsometry. The remaining slight disagreements in the parameters can be easily explained. The effect of the thin natural oxide layers of both $\mathrm{Si}$ and $\mathrm{Al}$ or the influence of imperfect adhesion on the pulses are not included. The speed of sound in the thin Al film used in the simulation is slightly lower than the literature values for bulk

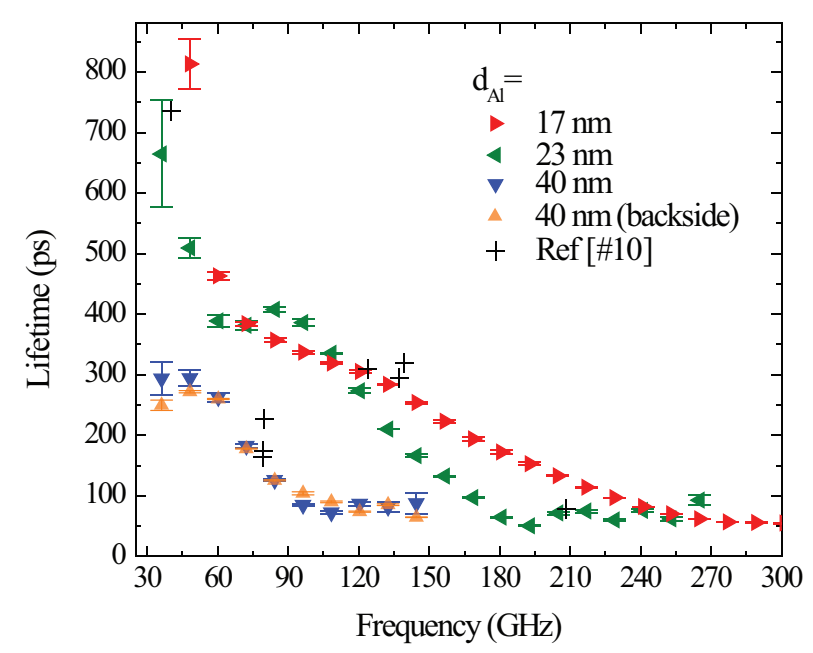

FIG. 10. (Color online) Lifetimes including error bars for varying phonon frequencies for different layer thicknesses.

crystalline $\mathrm{Al}$. We attribute this reduction to the polycrystalline structure of the $\mathrm{Al}$ film induced by the evaporation process.

\section{FREQUENCY-DEPENDENT PHONON LIFETIMES}

The system investigated allows us to measure the lifetimes of the coherent phonons in the two-layer system. Due to the fact that the pulses are well separated in the time domain, it is straightforward to extract frequency-dependent lifetimes for the coherent phonons generated in the system. By taking the FFT of every pulse and then plotting the amplitude of a specific frequency over the time delay for each pulse, the lifetime can be extracted by fitting an exponential to this curve for each desired frequency. The 9 ( $d_{\mathrm{Al}}=40 \mathrm{~nm}$ backside $), 12$ $\left(d_{\mathrm{Al}}=40 \mathrm{~nm}\right)$, and $13\left(d_{\mathrm{Al}}=17 \mathrm{~nm}\right.$ and $\left.d_{\mathrm{Al}}=23 \mathrm{~nm}\right)$ pulses were extracted from the time trace as described previously for the first three pulses. The phonon lifetimes and the error of the exponential fit for all the frequencies of the peaks of the frequency comb are plotted in Fig. 10. The lifetimes range from $800 \mathrm{ps}$ at $50 \mathrm{GHz}$ to $50 \mathrm{ps}$ at $300 \mathrm{GHz}$. The lifetimes for frequencies below $36 \mathrm{GHz}$ are not plotted due to the fact that they are longer than the measurement window, leading to a large error in the fit. Noteworthy is the lower lifetime by a factor of 2 for the sample with the thickest Al film. This is a very convenient measurement method to gain knowledge of the lifetimes for a layer system over a very broad frequency range. The large width of the frequency comb is thereby generated due to the small thickness of the metal layer already discussed by Thomsen et al. ${ }^{21}$ Due to the large number of pulses we obtain very small error bars for the exponential fit in contrast to previous measurements, where only up to three distinct pulses pulses could be measured. . $^{1,21}$

We want to point out that the lifetimes we are measuring are not intrinsic lifetimes. They are limited by extrinsic processes. Compared to the measurements of one to three times reflected 50 and $100 \mathrm{GHz}$ acoustic phonons in $50 \mu \mathrm{m}$ thick Si by Daly et al., giving lifetimes of 6.8 and $5.0 \mathrm{ns,}$ respectively, our results are one order of magnitude shorter. ${ }^{4}$ Recent experiments conducted with an ASOPS setup by Cuffe et al. have measured bare Si membranes, where the lifetimes 
for the fundamental modes - varied by changing the thickness of the membrane-were evaluated. ${ }^{10}$ Those results are also shown in Fig. 10. They are similar to our values, but correspond to both the thin and thick Al film samples. The shorter lifetimes compared to the lifetimes measured in thick Si plates were explained due to a large surface roughness dependence. The similarity of the results leads to the conclusion that a thin Al film does not change the damping properties for acoustic phonons in the $100 \mathrm{GHz}$ range too much. In contrast, for a thick Al film (40 nm instead of 17-23 nm) the lifetimes are shorter by a factor of 2 . We contribute this behavior to a stronger damping given through the polycrystalline structure of the $\mathrm{Al}$ film, but as the measurements by Cuffe indicate, this could also be due to a larger roughness. Further investigations are to be made to distinguish the influence of roughness from the polycrystalline structure.

Interestingly other possible effects of the used doublelayer system like additional surface roughness due to the polycrystalline film structure, adhesion properties of the two films, interface scattering, and the effect of inhomogeneous broadening of the acoustic pulse do not seem to play a large role. Therefore, once one is able to control the roughness, it should be possible to gain knowledge about these additional extrinsic processes and maybe also get insight on the intrinsic damping. Noteworthy is the plateau in the lifetimes for the 17 and $23 \mathrm{~nm}$ sample at around $100 \mathrm{GHz}$. For low frequencies the damping follows a $1 / \omega^{2}$ behavior, whereas for high frequencies it resembles a $1 / \omega^{4}$ behavior. The plateau lies in the intermediate regime of these two regions indicating a crossover of dominant damping processes.

\section{CONCLUSION}

In conclusion, we generated broadband acoustic frequency combs in the $100-\mathrm{GHz}$ frequency range over nearly an octave using Al/Si membranes. Nine to 45 well separated modes could be excited, given by the harmonics of the fundamental mode. A frequency shift of the higher harmonics due to the different mechanical properties of the two layers could be observed with great precision. The authors were able to detect up to 13 roundtrips of an acoustic pulse in the system. By comparing the individual reflected pulses it was possible to obtain the lifetimes for an $\mathrm{Al} / \mathrm{Si}$ layer system over the whole frequency range.

The origin of the stronger damping in the system compared to a thicker $\mathrm{Si}$ wafer, where the acoustic pulses are less frequently reflected, is still to be investigated. By comparing our measurements to measurements on bare $\mathrm{Si}$ membranes without an Al transducer, we can assume that the surface roughness plays an important role. Further investigations are needed to understand the frequency-dependent damping and to determine to which extent intrinsic Si damping plays a role, or if the system is entirely dominated by scattering at the interfaces. Also the influence of adhesion between $\mathrm{Al}$ and $\mathrm{Si}$ on the pulse propagation and comb properties has to be studied in more detail. This work is of relevance for the understanding of dissipation in nanomechanical and microelectromechanical systems in the frequency range well above $1 \mathrm{GHz}$. The used mechanism will be useful to study properties of thin membranes of other materials by using the double layer approach.

\section{ACKNOWLEDGMENTS}

This work was supported by the DFG through the SFB 767 (Germany) and by the Ministry of Science, Research and Arts of Baden-Württemberg (Germany). A. Bruchhausen thanks the Alexander von Humboldt Foundation (Bonn, Germany) for financial support. The authors also thank Matthias Hagner for providing support in the cleanroom. *martin.grossmann@uni-konstanz.de

${ }^{1}$ W. Chen and H. Maris, Philos. Mag. B 70, 687 (1994).

${ }^{2}$ G. A. Antonelli, B. Perrin, B. C. Daly, and D. G. Cahill, MRS Bull. 31, 607 (2006).

${ }^{3}$ A. Ward and D. A. Broido, Phys. Rev. B 81, 085205 (2010).

${ }^{4}$ B. C. Daly, K. Kang, Y. Wang, and D. G. Cahill, Phys. Rev. B 80, 174112 (2009).

${ }^{5}$ F. Hudert, A. Bruchhausen, D. Issenmann, O. Schecker, R. Waitz, A. Erbe, E. Scheer, T. Dekorsy, A. Mlayah, and J.-R. Huntzinger, Phys. Rev. B 79, 201307 (2009).

${ }^{6}$ A. Bruchhausen, R. Gebs, F. Hudert, D. Issenmann, G. Klatt, A. Bartels, O. Schecker, R. Waitz, A. Erbe, E. Scheer, J.-R. Huntzinger, A. Mlayah, and T. Dekorsy, Phys. Rev. Lett. 106, 077401 (2011).

${ }^{7}$ A. Bodapati, P. K. Schelling, S. R. Phillpot, and P. Keblinski, Phys. Rev. B 74, 245207 (2006).

${ }^{8}$ D. G. Cahill, W. K. Ford, K. E. Goodson, G. D. Mahan, A. Majumdar, H. J. Maris, R. Merlin, and S. R. Phillpot, J. Appl. Phys. 93, 793 (2003).
${ }^{9}$ A. A. Maznev, F. Hofmann, A. Jandl, K. Esfarjani, M. T. Bulsara, E. a. Fitzgerald, G. Chen, and K. A. Nelson, Appl. Phys. Lett. 102, 041901 (2013).

${ }^{10}$ J. Cuffe, O. Ristow, E. Chávez, A. Shchepetov, P.-O. Chapuis, F. Alzina, M. Hettich, M. Prunnila, J. Ahopelto, T. Dekorsy, and C. M. Sotomayor Torres, Phys. Rev. Lett. 110, 095503 (2013).

${ }^{11}$ T. Udem, R. Holzwarth, and T. W. Hänsch, Nature (London) 416, 233 (2002).

${ }^{12}$ N. D. Lanzillotti-Kimura, A. Fainstein, A. Huynh, B. Perrin, B. Jusserand, A. Miard, and A. Lemaître, Phys. Rev. Lett. 99, 217405 (2007)

${ }^{13}$ H. Sun, V. A. Stoica, M. Shtein, R. Clarke, and K. P. Pipe, Phys. Rev. Lett. 110, 086109 (2013).

${ }^{14}$ R. S. Bandhu, X. Zhang, R. Sooryakumar, and K. Bussmann, Phys. Rev. B 70, 075409 (2004).

${ }^{15}$ A. A. Savchenkov, A. B. Matsko, V. S. Ilchenko, D. Seidel, and L. Maleki, Opt. Lett. 36, 3338 (2011).

${ }^{16}$ T. J. Kippenberg, R. Holzwarth, and S. A. Diddams, Science (NY) 332, 555 (2011). 
${ }^{17}$ M. Hase, M. Katsuragawa, A. M. Constantinescu, and H. Petek, Nat. Photon. 6, 243 (2012).

${ }^{18}$ M. Bruel, B. Aspar, and A.-J. Auberton-Hervé, Jpn. J. Appl. Phys. 36, 1636 (1997).

${ }^{19}$ A. Bartels, R. Cerna, C. Kistner, A. Thoma, F. Hudert, C. Janke, and T. Dekorsy, Rev. Sci. Instrum. 78, 035107 (2007).

${ }^{20}$ M. A. Green and M. J. Keevers, Progr. Photovoltaics Res. Appl. 3, 189 (1995).

${ }^{21}$ C. Thomsen, H. T. Grahn, H. J. Maris, and J. Tauc, Phys. Rev. B 34, 4129 (1986).

${ }^{22}$ V. E. Gusev and O. B. Wright, Phys. Rev. B 57, 2878 (1998).
${ }^{23}$ S. A. Akhmanov and V. E. Gusev, Sov. Phys. Usp 35, 153 (1992). ${ }^{24}$ The amplitude of the reflectivity is given by $\left[Z_{\mathrm{Si}}-Z_{\mathrm{Al}}\right] /\left[Z_{\mathrm{Si}}+\right.$ $\left.Z_{\mathrm{Al}}\right]$, where $Z_{\mathrm{Si}, \mathrm{Al}}$ are the impedances of $\mathrm{Si}(19.6 \mathrm{GPa} \mathrm{s} / \mathrm{m})$ and $\mathrm{Al}$ $(17.0 \mathrm{GPa} \mathrm{s} / \mathrm{m})$, respectively .

${ }^{25}$ J. Groenen, F. Poinsotte, A. Zwick, C. M. Sotomayor Torres, M. Prunnila, and J. Ahopelto, Phys. Rev. B 77, 045420 (2008).

${ }^{26}$ E. J. Chern and H. T. C. Nielsen, J. Appl. Phys. 66, 2833 (1989).

${ }^{27}$ W. B. Gauster and D. H. Habing, Phys. Rev. Lett. 18, 1058 (1967).

${ }^{28}$ R. G. Stearns and G. S. Kino, Appl. Phys. Lett. 47, 1048 (1985).

${ }^{29}$ O. B. Wright and V. E. Gusev, Appl. Phys. Lett. 66, 1190 (1995). 